\title{
Less is more: the impact of maintenance treatment adherence in severe asthma clinical trials
}

\author{
Claire N. McBrien ${ }^{1}$ and Andrew Menzies-Gow ${ }^{2}$ \\ Affiliations: ${ }^{1}$ Imperial College London, London, UK. ${ }^{2}$ Respiratory Medicine, Royal Brompton Hospital, \\ London, UK. \\ Correspondence: A. Menzies-Gow, Royal Brompton Hospital, Respiratory Medicine, Sydney Street, London, \\ SW3 6NP, UK. E-mail: a.menzies-gowarbht.nhs.uk
}

@ERSpublications

Inadequate assessment of adherence to maintenance medication leads to loss of power and increased costs in trials of severe asthma therapy http://bit.ly/2USoiQU

Cite this article as: McBrien $\mathrm{CN}$, Menzies-Gow A. Less is more: the impact of maintenance treatment adherence in severe asthma clinical trials. Eur Respir J 2019; 53: 1900599 [https://doi.org/10.1183/ 13993003.00599-2019].

Asthma is the most common long-term respiratory condition, affecting more than 300 million people worldwide and, despite concerted efforts to achieve disease control and reduce exacerbations, it claims approximately 1000 lives each day [1]. The health and financial burdens associated with asthma are increasing, both at individual and population levels. A recent study estimated the annual cost of asthma in the USA to be more than USD 80 billion, taking into account direct medical costs, mortality and loss of attendance at school and work [2].

The pharmaceutical backbone of asthma management is maintenance treatment with inhaled corticosteroids (ICS). Treatment algorithms generally recommend low-dose ICS as early management, with stepwise dose escalation and the addition of long-acting $\beta_{2}$-agonists (LABAs) and other asthma controllers, such as leukotriene receptor antagonists and/or theophylline, if required [3]. A subgroup of people with asthma (around 5-10\%) either require a high-dose ICS/LABA inhaler plus other drugs in order to maintain asthma control, or remain uncontrolled despite such treatment [4]. In the most serious cases, treatment with oral corticosteroids (OCS) is required several times per year, or even on a daily basis, at which point add-on therapy with a biological agent is considered. The main goals of biological therapies, driven by the evidence from randomised controlled trials (RCTs), tend to be reductions in exacerbation frequency and OCS requirement, although improvements in spirometry and symptom scores can also often be achieved.

The costs of asthma correlate with disease severity, and primarily relate to direct outlays such as medications and hospitalisations [5]. Severe asthma therefore accounts for a disproportionately large fraction of spending on asthma. The full burden of OCS therapy is difficult to capture and quantify, but can include numerous unpleasant and costly short- and long-term adverse effects, including increased risk of osteoporosis, cardio/cerebrovascular disease, type 2 diabetes mellitus and weight gain [6]. The unmet need and high costs of managing severe asthma have lent support to the development of several biological therapies, which aim to improve disease control in patients with severe asthma when added to maximal conventional management [7]. 
A key component in the systematic assessment of severe asthma is the measurement of treatment adherence. Complete adherence to all prescribed medications (i.e. $100 \%$ of doses taken as directed) is generally recognised as an unrealistic aspiration; however, there is no consensus regarding the question "how much adherence is enough?" although figures of $70-80 \%$ are commonly used as cut-off points [8]. Crucially, there is no gold standard method for the measurement of adherence, with figures often generated using various information sources including direct patient questioning, prescription pick-up rates and inhaler dose counters [9]. More recently, inhaler devices designed for objective remote adherence monitoring have been developed and fractional exhaled nitric oxide suppression tests have been suggested as a method of discerning "adherent" from "non-adherent" subgroups [10, 11]. Phase III RCTs of biological therapies for asthma typically recruit subjects who, despite being prescribed high-dose ICS/ LABA and sometimes frequent OCS courses or maintenance OCS, remain symptomatic with ongoing exacerbations and evidence of type 2 high airway inflammation. These inclusion criteria have a direct impact on drug licensing and payer decisions, which are based on a combination of clinical efficacy and cost-effectiveness. Patients within this cohort may have severe asthma with relative resistance to glucocorticoids or alternatively be poorly adherent with conventional therapy, or indeed be both. It is frequently difficult to determine the proportion of these cohorts that are represented in phase III studies, as adherence to conventional treatment is rarely consistently measured either at baseline or during the course of treatment with the drug/placebo. It is probable that improved adherence with conventional asthma therapy during RCTs is one of the key contributing factors to the marked improvements frequently observed in placebo groups [12-15]. This effect is likely to be especially relevant in healthcare economies where the provision of standard of care treatment at no cost to participants in clinical trials removes the financial barrier to adherence. The magnitude of the placebo effect in these studies has led to the requirement for increased subject numbers to ensure that they are adequately powered for the expected effect of the novel therapy on annualised exacerbation frequency.

In this issue of the European Respiratory Journal, Мокока et al. [16] take a pragmatic approach to the issue of (unrecorded) variable adherence in trials of add-on therapy for severe asthma, aiming to quantify the influence of adherence on the variance of clinical outcomes, and modelling the extent to which power could be enhanced by adequately accounting for adherence as a confounder. This is an admirable objective, especially given the lack of adherence data supplied by such trials, and is supported by an elegant statistical approach, which aims to avoid the pitfalls of uncompromising "all-or-nothing" definitions of adherence.

The authors' comprehensive literature search identified 87 relevant RCTs, only 20 (23\%) of which reported adherence data and none of which provided objective evidence of adherence. Although annualised exacerbation frequency tends to be the primary outcome in most trials of add-on therapy for severe asthma, due to the technical requirements of their model, the authors chose to focus on the forced expiratory volume in $1 \mathrm{~s}\left(\mathrm{FEV}_{1}\right)$ as a more standardised and comparable measure across the various studies. While understandable, this departure from the conventional primary outcome is unfortunate as the exacerbation rate is both clinically important and a high priority for payers.

20 RCTs that reported FEV1 as an absolute value in litres underwent further analysis. Adherence to conventional treatment was reported in half of these studies. The pooled variance in FEV1 was significantly lower in trials that reported adherence compared to those that did not $\left(S^{2}=0 \cdot 144 L^{2}\right.$ versus $S^{2}=0 \cdot 168 L^{2}$ ). The reduced variance in trials that reported adherence resulted in an improved mean power to detect a $100 \mathrm{~mL}$ change in $\mathrm{FEV}_{1}$ (59\% versus 49\%), despite a smaller mean sample size. The authors assert that in the studies that did not report adherence, a significant component of the variance in FEV1 may be attributed to variations in adherence, but correctly identify the possibility that other differences in study methodology or execution had some impact.

When confronted with data suggestive of suboptimal adherence to conventional asthma treatment, even among patients with severe disease selected for clinical trials of add-on therapies, the temptation is to focus purely on optimising adherence and excluding "non-adherent" participants from RCTs. In that scenario, it would be reasonable to expect that the very act of identifying and improving adherence to conventional treatment may improve symptom control and exacerbation frequency such that the pool of patients who require add-on therapy is reduced, and subjects who continue to suffer poor disease control would be more likely to demonstrate a clinically significant improvement with the study drug when compared with placebo.

We should resist the urge to completely eradicate normal human behaviour from RCTs. Treatment adherence is a complex and multifactorial entity, and it is important to acknowledge the somewhat limited capacity to accurately assess and persistently improve adherence in a real-world scenario. In their article, Мокока et al. [16] make a compelling argument that a more sensible approach is to recognise variable 
adherence as a potential confounder in RCTs, and to objectively monitor and correct for it, thus ensuring results remain clinically translatable. Although this added rigour would increase per-subject trial costs (and perhaps diminish the attractiveness of RCT participation for some individuals), application of a model to correct for adherence variations indicated that sample sizes could be reduced by around $50 \%$, suggesting considerable financial savings could be made.

Another factor to consider, which may limit the potential to perform smaller phase III studies, is the valuable contribution of phase III clinical trials to the characterisation of the safety profile of new drugs in relevant patient populations. Even if the power to detect clinical efficacy can be significantly enhanced, we should be cautious about drastically reducing recruitment targets for RCTs, lest we blunt our ability to detect drug safety signals prior to licensing decisions and widespread clinical use.

The clinical imperative to develop new and better treatments for patients with severe asthma is clear. Approaches to maximise the power of RCTs and therefore reduce the numbers of subjects who must be recruited are attractive. Мокока et al. [16] address the issue of accounting for adherence in trials of severe asthma therapy and suggest that objectively monitoring and adjusting for variable adherence levels in future studies could improve power such that sample sizes and costs could be reduced considerably. This message should be interpreted carefully as it may not be directly applicable to the commonest primary outcome (annualised exacerbation rates) and large-scale phase III trials generate other important data regarding safety and secondary clinical outcomes.

Conflict of interest: C.N. McBrien has attended an international conference with Boehringer Ingelheim and a national meeting with TEVA. A. Menzies-Gow reports grants, personal fees, non-financial support and other (research conducted in the author's institution and consultancy agreement) from AstraZeneca, personal fees from Novartis, personal fees and non-financial support from Teva, personal fees and other (consultancy agreement) from Vectura, personal fees and other (research conducted in the author's institution) from GlaxoSmithKline, personal fees, non-financial support and other (research conducted in the author's institution) from Boehringer Ingelheim, personal fees and other (consultancy agreement) from Sanofi, and personal fees from Hoffman La Roche, outside the submitted work.

\section{References}

1 Global, regional, and national deaths, prevalence, disability-adjusted life years, and years lived with disability for chronic obstructive pulmonary disease and asthma, 1990-2015: a systematic analysis for the Global Burden of Disease Study 2015. Lancet Respir Med 2017; 5: 691-706.

2 Nurmagambetov T, Kuwahara R, Garbe P. The economic burden of asthma in the United States, 2008-2013. Ann Am Thorac Soc 2018; 15: 348-356.

3 Global Initiative for Asthma. Global Strategy for Asthma Management and Prevention, 2018. Available from www. ginasthma.org

4 Chung KF, Wenzel SE, Brozek JL, et al. International ERS/ATS guidelines on definition, evaluation and treatment of severe asthma. Eur Respir J 2014; 43: 343-373.

5 Bahadori K, Doyle-Waters MM, Marra C, et al. Economic burden of asthma: a systematic review. BMC Pulm Med 2009; 9: 24.

6 Price DB, Trudo F, Voorham J, et al. Adverse outcomes from initiation of systemic corticosteroids for asthma: long-term observational study. J Asthma Allergy 2018; 11: 193-204.

$7 \quad$ McCracken JL, Tripple JW, Calhoun WJ. Biologic therapy in the management of asthma. Curr Opin Allergy Clin Immunol 2016; 16: 375-382.

8 Barnes CB, Ulrik CS. Asthma and adherence to inhaled corticosteroids: current status and future perspectives. Respir Care 2015; 60: 455-468.

9 Lindsay JT, Heaney LG. Non-adherence in difficult asthma and advances in detection. Expert Rev Respir Med 2013; 7: 607-614.

10 Bonini M, Usmani OS. Novel methods for device and adherence monitoring in asthma. Curr Opin Pulm Med 2018; 24: 63-69.

11 Heaney LG, Busby J, Bradding P, et al. Remotely monitored therapy and nitric oxide suppression identifies nonadherence in severe asthma. Am J Respir Crit Care Med 2019; 199: 454-464.

12 Humbert M, Beasley R, Ayres J, et al. Benefits of omalizumab as add-on therapy in patients with severe persistent asthma who are inadequately controlled despite best available therapy (GINA 2002 step 4 treatment): INNOVATE. Allergy 2005; 60: 309-316.

13 Pavord ID, Korn S, Howarth P, et al. Mepolizumab for severe eosinophilic asthma (DREAM): a multicentre, double-blind, placebo-controlled trial. Lancet 2012; 380: 651-659.

14 Castro M, Zangrilli J, Wechsler ME, et al. Reslizumab for inadequately controlled asthma with elevated blood eosinophil counts: results from two multicentre, parallel, double-blind, randomised, placebo-controlled, phase 3 trials. Lancet Respir Med 2015; 3: 355-366.

15 Bleecker ER, FitzGerald JM, Chanez P, et al. Efficacy and safety of benralizumab for patients with severe asthma uncontrolled with high-dosage inhaled corticosteroids and long-acting beta2-agonists (SIROCCO): a randomised, multicentre, placebo-controlled phase 3 trial. Lancet 2016; 388: 2115-2127.

16 Mokoka MC, McDonnell MJ, MacHale E, et al. Inadequate assessment of adherence to maintenance medication leads to loss of power and increased costs in trials of severe asthma therapy: results from a systematic literature review and modelling study. Eur Respir J 2019; 53: 1802161. 